\title{
Resolution of the Electron Microscope at the Atomic Scale
}

\author{
Michael A. O'Keefe ${ }^{1}$, Lawrence F. Allard ${ }^{2}$, Douglas A. Blom ${ }^{3}$ \\ ${ }^{1}$ OKCS, 18528 Mesa Verde Way, Castro Valley, CA 94552 USA \\ ${ }^{2}$ Materials Science \& Technology Div., Oak Ridge National Laboratory, Oak Ridge, TN 37831 USA \\ ${ }^{3}$ EM Center, University of South Carolina, 715 Sumter St, Columbia, SC 29208 USA
}

The importance of atomic-resolution electron microscopy as a tool for structure analysis lies in its ability to produce images in which each peak corresponds to the position of an atom (or atomic column) within the specimen. Being able to distinguish between atoms (or columns) that appear close together when projected in the chosen viewing direction depends on the resolution of the microscope. Knowledge of the resolution of any particular electron microscope is crucial to judge if its resolution is appropriate for the specimen. In addition, resolution quality will determine the precision of measured atom positions.

Rayleigh's resolution criterion, an accepted standard in optics, was derived as a means for judging when two sources of light (stars) were distinguishable from a single source by their image peaks [1]. At the atomic level, resolution in the electron microscope is demonstrated absolutely and canonically in real space by separation of image peaks representing atoms. We have proposed an "A-OK" set of test specimens that can evaluate resolutions from $1.6 \AA$ to $0.5 \AA$ unambiguously in real space $[2,3]$, and we have shown how atom positions within the specimen can be determined from image peaks by including image resolution quality $[3,4]$. Real-space measurements of resolution bypass the different imaging modalities used by TEM and STEM and apply to both. In the TEM, interference of electron waves from the specimen images the relative phases of the waves as peaks that map the atom positions and scattering power. Resolution of $|\mathbf{d}|$ is achieved when atoms separated by a (projected) distance $|\mathbf{d}|$ can be perceived as separate objects. Resolution $|\mathbf{d}|$ requires the presence of spatial frequency $1 /|\mathbf{d}|$ in the TEM image spectrum, but mere presence of the $1 /|\mathbf{d}|$ frequency is not sufficient to demonstrate a resolution of $|\mathbf{d}|$ [5]. In the STEM, a probe size of $|\mathbf{d}|$ is a necessary, but not sufficient, requirement to achieve a resolution of $|\mathbf{d}|$ [6]. We propose that both TEM and STEM use the same standard of resolution - separation of atom peaks in the image. Peak positions will be accurate under Rayleigh conditions with a $25 \%$ dip between them, and deviate progressively as resolution moves towards the Sparrow criterion with zero dip [3]. Atoms are still clearly resolved even at sub-Rayleigh resolution $[3,6]$. An example from a silicon specimen imaged in [112] orientation shows clear $0.78 \AA$ resolution although peak separation does not attain Rayleigh conditions (fig. 1) [7].

In spite of clear and definite quantification of resolution in real space, there exists a tendency to try to gauge resolution in reciprocal space. A diffractogram from an electron microscope image will show all spatial frequencies contained in the image, but will not distinguish between those containing specimen information and those generated by the imaging process. Even when random noise is accounted for by the use of Young's interference fringes (YIFs), image frequencies can extend to higher frequencies than the structural information coming from the specimen. For HRTEMs, resolution can be approximated in reciprocal space using a diffractogram from an amorphous specimen imaged at Scherzer defocus (look for the cross-over), but determination of the HRTEM information limit (the upper bound of resolution for a corrected HRTEM), is problematic using Young's fringes. We have demonstrated that YIFs will extend beyond the microscope information limit. Although this effect can be minimized for thin amorphous specimens, it becomes dominant when Thon rings are not visible, leading to incorrect overoptimistic estimates of YIF limit as the TEM information limit. In fact, as we have pointed out, the two limits are independent. The information limit of the TEM is set by the temporal coherence spread of 
focus, whereas the Young's fringe limit is set by the spatial coherence incident beam convergence $[7,8]$. Second-order interferences [5] allow Young's fringes in diffractograms to extend up to twice the linear information limit (the resolution limit of a Cs-corrected TEM) unless they are constrained by blurring factors such as detector point-spread function or instrument vibration. Experimental results confirm that Young's fringes routinely extend past information limits $[8,9,10]$.

An upper limit to electron image resolution is set by the specimen. Electrons scatter from specimen potential in which atom widths appear as intrinsic atom size broadened by vibrational displacements due to temperature. Calculations for silicon in [116] orientation show that atoms in images taken at room temperature would not achieve Rayleigh resolution due solely to the room-temperature temperaturefactor for silicon of $0.467 \AA^{2}$ no matter how good the microscope resolution $[3,11,12]$.

[1] Lord Rayleigh, Philosophical Magazine 47, 310: (1874) 81-93.

[2] M. A. O'Keefe, L.F. Allard \& D. A. Blom, Microscopy \& Microanalysis 10 S02 (2004) 1002-1003.

[3] M. A. O'Keefe, L.F. Allard \& D. A. Blom, J. Electron Microscopy 54 (2005) 169-180.

[4] M. A. O'Keefe, L.F. Allard \& D. A. Blom, Microscopy \& Microanalysis 11 S02 (2005) 540-541.

[5] M.A. O'Keefe, 37th Ann. Proc. EMSA, San Antonio, Texas (1979) 556-557.

[6] Y. Peng, M. P. Oxley, A. R. Lupini et al, Microsc Microanal 14(1) (2008) 36-47.

[7] M.A. O'Keefe, Ultramicroscopy 108 (2008) 196-209.

[8] M. A. O’Keefe, L.F. Allard \& D. A. Blom, Microscopy \& Microanalysis 15 S02 (2009) 1470-1471.

[9] M. A. O’Keefe, L.F. Allard \& D. A. Blom, Microscopy \& Microanalysis 16 S02 (2010) 766-767.

[10] A. Genç et al, Microscopy \& Microanalysis 12 S02 (2006) 1478-1479.

[11] M. A. O'Keefe, L.F. Allard \& D. A. Blom, Microscopy \& Microanalysis 13 S02 (2007) 766-767.

[12] Research at Oak Ridge National Laboratory's High Temperature Materials Laboratory sponsored by U.S.

Department of Energy, Office of Energy Efficiency \& Renewable Energy, Vehicle Technologies program.

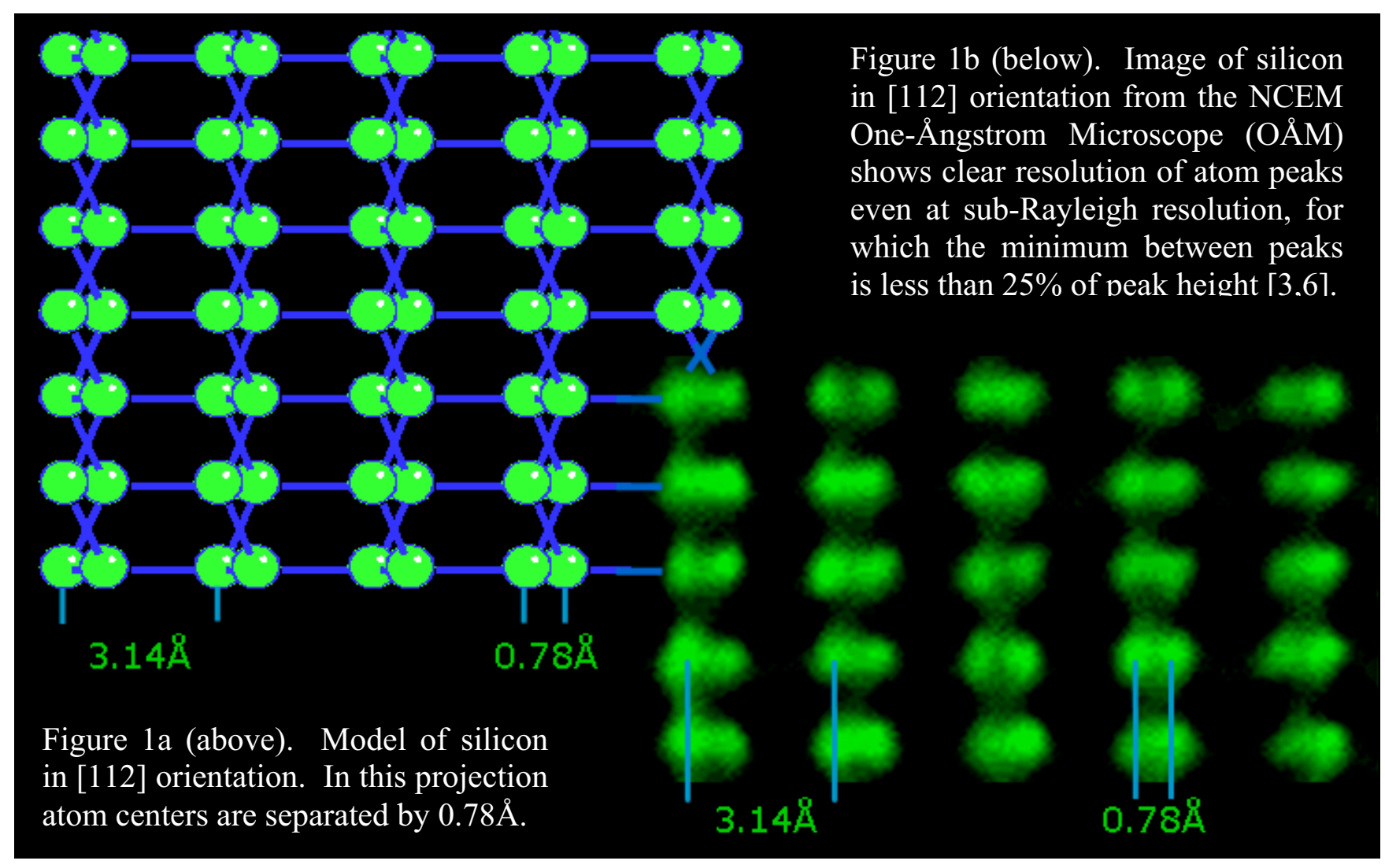

